\title{
Rising Incidence of Neuroendocrine Tumors in Singapore: An Epidemiological Study
}

\author{
Prajwala S Prakash ${ }^{1}$, Sujith Wijerathne ${ }^{2}$, Rajeev Parameswaran ${ }^{3}$
}

\begin{abstract}
Aim: The incidence of neuroendocrine tumors (NETs) is increasing worldwide. This study presents the epidemiological trends of NETs and survival results from Singapore.

Materials and methods: This is a retrospective population-based study of all NETs treated during 1993-2014 in Singapore. The primary outcome studied was trends in incidence of NETs, and secondary outcomes were gender, ethnic and histological variations, and overall survival results. Results: During the study period, 1,725 cases were identified and the age-standardized incidence increased from 0.8 to 3 per 100,000 per year. The mean age of diagnosis was $56.0( \pm 25.5)$ years, with the highest incidence among ethnic Chinese $(n=1391,80.6 \%)$ and gender ratio nearly 1:1. Histologically, the tumors were predominantly carcinoid tumor (52.6\%) and neuroendocrine carcinoma (40.3\%), with tumors arising most commonly in the rectum (26.0\%), colon (13.7\%), lung and bronchus (13.7\%), pancreas (9.4\%), stomach (7.1\%), small intestine (5.9\%), and other organs (24.1\%). Majority were G1 (51.9\%) and G3 (33.9\%) tumors, and of clinical stage I (41.0\%) and stage IV (35.9\%). The most commonly rendered treatment was surgery alone (36.2\%). The 5-year overall survival for the cohort for all NETs was 38.1\%, and 10 -year survival was $22.0 \%$. Predictors for poor survival in this cohort include primary tumor site, gender, and advancing age.

Conclusion and clinical significance: The incidence of NETs in Singapore has markedly increased nearly fourfold over the last two decades, possibly due to improved detection. However, there are various other unevaluated factors that warrant further investigation.

Keywords: Carcinoid, Cohort study, Epidemiology, Neuroendocrine tumor.

World Journal of Endocrine Surgery (2020): 10.5005/jp-journals-10002-1280
\end{abstract}

\section{INTRODUCTION}

Neuroendocrine tumors (NETs), which were formerly known as carcinoid tumors, are considered to be rare and this has resulted in scarcity of data on these tumors specific to countries and regions. The indolent nature and atypical presentations of these tumors also contribute to delay in diagnosis and treatment. However, there has been an observed increase in the incidence of these tumors over the past few decades ${ }^{1-3}$ but an obvious cause for this increase has not been identified.

The increased incidence of these NETs has been reported from all over the world. The age-adjusted incidence of gastroenteropancreatic NETs has increased 3.65 times in the United States and 4.8 times in the United Kingdom over a period of four decades (1973-2007). ${ }^{4}$ In England, the overall incidence has increased from 0.27 (per 100,000 per year) to 1.32 for men and from 0.35 to 1.33 for women during a similar time period of four decades. ${ }^{5}$ Data from the Surveillance, Epidemiology, and End Results (SEER) program in the United States show that the reported annual age-adjusted incidence of NETs has increased from 1.09 (per 100,000) in 1973 to 5.25 (per 100,000) in 2004. ${ }^{6}$ Similar increase in incidence for NETs has been reported from rest of the Europe $\mathrm{e}^{7-11}$ and North America ${ }^{2}$ as well. Epidemiological data on NETs from Asia are limited but those available from Japan ${ }^{12,13}$ and Taiwan ${ }^{14}$ have shown similar increment pattern in the incidence as seen in rest of the world.

The rise in the incidence of NETs could be due to a true increase in the disease prevalence or due to increased number of cases diagnosed as a result of improved imaging and diagnostics. Pancreatic NETs are an example for those NETs increasingly discovered during radiologic or endoscopic examinations; however, the frequency of incidental detection of these tumors is unknown. ${ }^{15}$
${ }^{1-3}$ Division of Endocrine Surgery, National University Hospital, Singapore Corresponding Author: Rajeev Parameswaran, Division of Endocrine Surgery, National University Hospital, Singapore, Phone: +65 6779 5555, e-mail: rajeev_parameswaran@nuhs.edu.sg

How to cite this article: Prakash PS, Wijerathne S, Parameswaran R. Rising Incidence of Neuroendocrine Tumors in Singapore: An Epidemiological Study. World J Endoc Surg 2020;12(1):1-4.

Source of support: Nil

Conflict of interest: None

The clinical and pathological heterogeneous nature of these tumors together with the trends in age, gender, geographical distribution, and obscure symptoms that they possess and the variable degree of survival that has shown to depend on the site, grade, clinical stage, and the difference in response to multiple treatment modalities can be recognized as important factors ${ }^{16}$ in this disease spectrum that needs more attention and understanding in order to optimize diagnosis and management of NETs. The aim of our study was to investigate the time trends in the incidence of thyroid cancer in Singapore from 1993 to 2014 using the National Cancer Registry. We also looked at the trends in pathology, treatments, mortality, and ethnic differences, as Singapore has a population made up of various ethnicities.

\section{Materials and Methods \\ Study Design}

This is a retrospective population-based study to investigate the trends in incidence, gender, ethnic and histological variations, and overall survival for NETs in Singapore over a period of 22 years.

(-) The Author(s). 2020 Open Access This article is distributed under the terms of the Creative Commons Attribution 4.0 International License (https://creativecommons. org/licenses/by-nc/4.0/), which permits unrestricted use, distribution, and non-commercial reproduction in any medium, provided you give appropriate credit to the original author(s) and the source, provide a link to the Creative Commons license, and indicate if changes were made. The Creative Commons Public Domain Dedication waiver (http://creativecommons.org/publicdomain/zero/1.0/) applies to the data made available in this article, unless otherwise stated. 
Data were obtained from the Singaporean national database owned by the local Ministry of Health. This database covered a Singaporean population of approximately 4 million individuals in the early 1990 s up to mid-2000s and a larger population of roughly 5 million people beyond the mid-2000s.

All cases of NETs treated during the period, January 1, 1993 to December 31, 2014, in Singapore and with complete clinical data have been included in this study. Cases of NETs with incomplete clinical data such as undocumented histological type, grade, and stage of tumor have been excluded from this study.

\section{Data Collection}

Demographic data, including patient age, ethnicity, and gender, as well as clinical data such as primary tumor site, histological type, grade and stage of NET, and treatment rendered were collected. Primary tumor site and histological type were coded as per the ICD-9 diagnostic code descriptions. For histological type, the first four digits of the code indicate the specific histological term, and the fifth digit after the slash is the behavior code. The project was approved by the Institutional Research Board (DSRB:2014/00466).

\section{Statistical Analysis}

The primary outcome studied was the trend in age-standardized incidence rates over the study period of 22 years. This was calculated using the World Health Organization (WHO) standard population.

IBM SPSS Statistics 23 was used for statistical analysis. Continuous variables were expressed as mean and standard deviation (SD). The basic statistical analysis was performed for categorical variables in the form of row percentages.

The secondary outcomes studied were the gender, ethnic and histological variations, and overall survival at 5 years and at 10 years. Posttreatment survival analysis was performed using the KaplanMeier estimator and a survival curve was generated.

\section{Results}

A total of 1,725 cases of NETs were identified for inclusion in our study during the 22-year period of January 1, 1993 to December 31, 2014. There were 918 males (53.2\%) and 807 (46.8\%) females in our study, with an almost equal gender ratio of 1.14:1.

The age at diagnosis ranged from 19 to 82 years old. The mean age of diagnosis was $56.0( \pm 25.5)$, with the highest proportion (32.9\%) being age at diagnosis under 50 years old.

There are four official ethnic groups in Singapore-namely, the Chinese, Malay, Indian, and others. The highest incidence of NETs was among the Chinese (80.6\%).

The aforementioned demographic data are presented in Table 1.

As seen in Table 2, the age-standardized incidence of NETs demonstrated an overall increasing trend in the study period. The lowest age-standardized incidence in the 22 -year period in question was 0.8 per 100,000 in the year 1993 and reached its peak of 3.0 per 100,000 in the year 2013. This represents an almost fourfold increase in the age-standardized incidence rate. The age-standardized incidence in the final of the 22 years studied, that is, in the year 2014, was 2.4 per 100,000.

The clinical profile of the NETs in our study, such as primary site, histological type, grade, and stage, has been depicted in Table 3 and described below.

In terms of primary tumor site, tumors most commonly arose in the rectum (26.0\%), colon (13.7\%), lung and bronchus (13.7\%), pancreas $(9.4 \%)$, stomach $(7.1 \%)$, small intestine $(5.9 \%)$, and other
Table 1: Demographic data of study population

\begin{tabular}{ll}
\hline Age (years) & Number $(\%)$ \\
\hline $0-49$ & $567(32.9)$ \\
$50-59$ & $410(23.8)$ \\
$60-69$ & $385(22.3)$ \\
$70-79$ & $282(16.3)$ \\
$80+$ & $81(4.7)$ \\
Mean (SD) & $56.01( \pm 25.5)$ \\
\hline Ethnicity & Number $(\%)$ \\
\hline Chinese & $1,391(80.6)$ \\
Malay & $151(8.8)$ \\
Indian & $139(8.1)$ \\
Others & $44(2.6)$ \\
\hline Gender & Number $(\%)$ \\
\hline Male & $918(53.2)$ \\
Female & $807(46.8)$ \\
\hline
\end{tabular}

Table 2: Annual incidence and age-standardized incidence rate (ASR) of NETs in Singapore between 1993 and 2014

\begin{tabular}{lcl}
\hline Year of diagnosis & Number (\%) & ASR \\
\hline 1993 & $24(1.4)$ & 0.8 \\
1994 & $29(1.7)$ & 0.9 \\
1995 & $32(1.9)$ & 1.1 \\
1996 & $30(1.7)$ & 0.9 \\
1997 & $35(2.0)$ & 1.1 \\
1998 & $41(2.4)$ & 1.3 \\
1999 & $54(3.1)$ & 1.5 \\
2000 & $60(3.5)$ & 1.6 \\
2001 & $65(3.8)$ & 1.7 \\
2002 & $61(3.5)$ & 1.5 \\
2003 & $57(3.3)$ & 1.5 \\
2004 & $69(4.0)$ & 1.7 \\
2005 & $69(4.0)$ & 1.6 \\
2006 & $90(5.2)$ & 2.0 \\
2007 & $96(5.6)$ & 2.0 \\
2008 & $101(5.9)$ & 2.1 \\
2009 & $124(7.2)$ & 2.4 \\
2010 & $128(7.4)$ & 2.6 \\
2011 & $106(6.1)$ & 2.0 \\
2012 & $151(8.8)$ & 2.9 \\
2013 & $166(9.6)$ & 3.0 \\
2014 & $137(7.9)$ & 2.4 \\
\hline & & \\
\hline
\end{tabular}

organs (24.1\%). Histologically, the tumors were predominantly carcinoid tumors (52.6\%) and neuroendocrine carcinomas (40.3\%).

The pathological grading of tumors was in accordance with the 2010 WHO classification scheme for neuroendocrine neoplasms. ${ }^{17}$ Majority of NETs in this study were NET G1 (51.9\%), followed by NET G3 (33.9\%). The most common clinical stage for patients in our study was stage I disease (41.0\%), and the second most common was final stage or stage IV disease (35.9\%).

Table 4 shows the numerical and percentage breakdown of treatment(s) that were offered to patients in the study. The treatment options were radiotherapy, chemotherapy, or surgeryeach in isolation as a monotherapy or in various combinations as 
Table 3: Clinical profile of NETs in study population

\begin{tabular}{|c|c|c|}
\hline Histological type & Code(s) & Number (\%) \\
\hline Islet cell carcinoma & $8150 / 1,8150 / 3$ & $12(0.7)$ \\
\hline Insulinoma & $8151 / 3$ & $3(0.2)$ \\
\hline Glucagonoma & $8152 / 3$ & $0(0.0)$ \\
\hline Gastrinoma & $8153 / 1$ & $3(0.2)$ \\
\hline $\begin{array}{l}\text { Mixed islet cell and exocrine } \\
\text { adenoma }\end{array}$ & $8154 / 3$ & $4(0.2)$ \\
\hline VIPoma & $8155 / 3$ & $0(0.0)$ \\
\hline Somatostatinoma & $8156 / 3$ & $0(0.0)$ \\
\hline Enteroglucagonoma & $8157 / 3$ & $0(0.0)$ \\
\hline Multiple endocrine adenomas & $8360 / 1$ & $0(0.0)$ \\
\hline Merkel cell cancer & $8247 / 3$ & $16(0.9)$ \\
\hline $\begin{array}{l}\text { Medullary carcinoma with } \\
\text { amyloid stroma }\end{array}$ & $8345 / 3$ & $59(3.4)$ \\
\hline Pheochromocytoma & $8700 / 3$ & $15(0.9)$ \\
\hline Carcinoid tumor & $\begin{array}{l}8240 / 1,8240 / 3 \\
8241 / 3,8242 / 3 \\
8243 / 3,8244 / 3 \\
8245 / 1,8245 / 3 \\
8249 / 3\end{array}$ & $908(52.6)$ \\
\hline Neuroendocrine carcinoma & $\begin{array}{l}8246 / 3,8013 / 3 \\
8574 / 3\end{array}$ & $695(40.3)$ \\
\hline Sympathetic paraganglioma & $8681 / 1$ & $0(0.0)$ \\
\hline Parasympathetic paraganglioma & $8682 / 1$ & $0(0.0)$ \\
\hline Paraganglioma, malignant & $8680 / 3$ & $3(0.2)$ \\
\hline Glomus jugulare tumor, NOS & $8690 / 1$ & $7(0.4)$ \\
\hline Primary site (ICD-9) & & Number (\%) \\
\hline Lung and bronchus (162) & & $236(13.7)$ \\
\hline Small intestine (152) & & $102(5.9)$ \\
\hline Rectum (154) & & $449(26.0)$ \\
\hline Colon (153) & & $238(13.7)$ \\
\hline Stomach (151) & & $122(7.1)$ \\
\hline Pancreas (157) & & $162(9.4)$ \\
\hline Others/unknown & & $416(24.1)$ \\
\hline Grade/differentiation & & Number (\%) \\
\hline 1 & & $334(51.9)$ \\
\hline II & & $77(12.0)$ \\
\hline III & & $218(33.9)$ \\
\hline IV & & $14(2.2)$ \\
\hline Clinical stage & & Number (\%) \\
\hline I & & $322(41.0)$ \\
\hline II & & $86(10.9)$ \\
\hline III & & $96(12.2)$ \\
\hline IV & & $282(35.9)$ \\
\hline
\end{tabular}

multimodal therapeutic approaches. The most commonly rendered treatment was surgery alone (36.2\%).

As censored on December 31, 2015, the 5-year overall survival for the cohort for all NETs was $38.1 \%$, and 10 -year survival was $22.0 \%$. These survival analysis data are portrayed in both Table 5 and the Kaplan-Meier curve in Figure 1. Predictors for poor survival in this cohort include primary tumor site, gender, and advancing age.

\section{Discussion}

To our knowledge, our study is the first Southeast Asian study to report epidemiological data on NETs, enabling us to present a new
Table 4: Treatment(s) offered to study population

\begin{tabular}{lc}
\hline Treatment & Number (\%) \\
\hline S only & $625(36.2)$ \\
S + R only & $15(0.9)$ \\
S + C only & $57(3.3)$ \\
S + R + C only & $21(1.2)$ \\
R only & $41(2.4)$ \\
C only & $86(5.0)$ \\
R + C only & $57(3.3)$ \\
All other combinations & $86(5.0)$ \\
Unknown & $737(42.7)$ \\
\hline
\end{tabular}

$S$, surgery; $R$, radiotherapy; $C$, chemotherapy

Table 5: Overall survival data of study population

\begin{tabular}{ll}
\hline Survival & Number (\%) \\
\hline$<1$ & $375(21.7)$ \\
$1-5$ & $657(38.1)$ \\
$>5-10$ & $379(22.0)$ \\
$>10-15$ & $168(9.8)$ \\
$>15-20$ & $97(5.6)$ \\
$>20$ & $49(2.8)$ \\
\hline
\end{tabular}

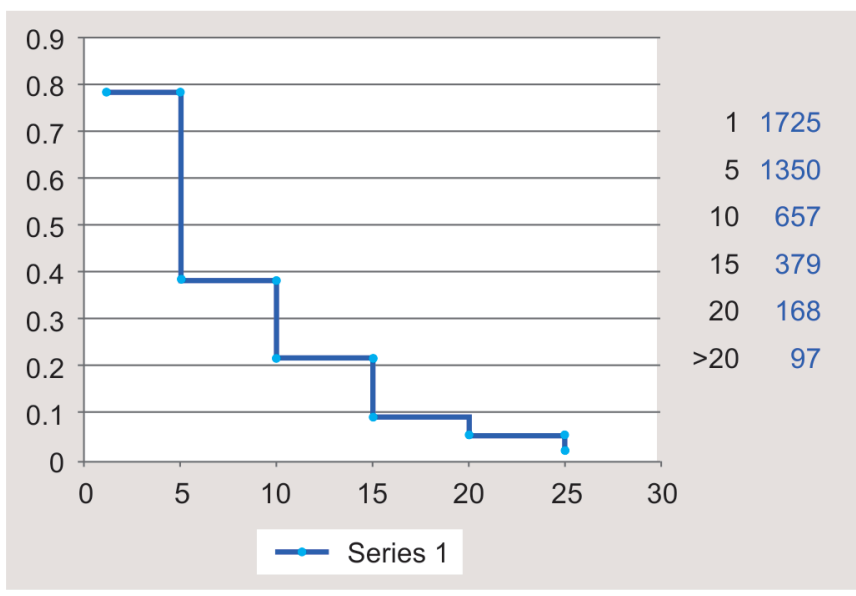

Fig. 1: Kaplan-Meier survival curve for study population

perspective amidst the existing North American, European, and East Asian literature. Furthermore, being a large population-based study spanning more than two decades improves the accuracy and reliability of our results.

The primary outcome studied was the trend in age-standardized incidence rates and based on our data, an almost fourfold increase in the age-standardized incidence rate was observed over the last two decades or so. This result of increasing incidence rates is consistent with findings from international epidemiological studies from Europe, North America, and other parts of Asia. ${ }^{10}$

In keeping with postulations in the literature, it is likely that the increased age-standardized incidence rates of NETs in Singapore can be attributed to multiple factors. First, at a semantic level, there has been a global shift in the pathological classification of such tumors from "other entities" like undifferentiated carcinoma to a formal entity called NETs. ${ }^{10}$

In addition, more sophisticated diagnostic tools-including radiological, endoscopic, ${ }^{9,13,15}$ and immune-histochemical 
techniques ${ }^{10}$ - are now available. Also, diagnostic facilities have become more accessible to the Singaporean population due to increasing government subsidies for health care, and a local example would be subsidized colorectal cancer screening programs with endoscopy.

However, the absolute age-standardized incidence rates in our study are almost twice those reported by a Taiwanese national registry-based study covering a reasonably similar period of January 1, 1996 to December 31, 2008, and a total of 2,187 cases. $^{14}$ The aforementioned study presented that the age-standardized incidence rate of NETs increased from 0.30 per 100,000 in 1996 to 1.51 per 100,000 in 2008, as opposed to our current results of 0.80 per 100,000 in 1993 to a peak rate of 3.0 per 100,000 in 2013.

Our incidence results mirror those from Caucasian populations (2.5-5 cases per 100,000 in North America) ${ }^{3}$ in spite of the fact that the Singaporean population is nearly $76 \%$ Chinese and theoretically should have resembled the Taiwanese population instead. This is indeed a noteworthy finding that begs the question as to whether the ethnic minorities in Singapore, that is, the Malays, Indians, and others, contribute at a disproportionately higher level to the incidence rates than their actual percentage make-up within the Singaporean population.

This also leads us to a secondary outcome studied-ethnic variations. Our data have even more interestingly shown that, in fact, the ethnic Chinese population in Singapore made up $80.6 \%$ of all patients with NETs in our study. This is commensurate with their representation within our population. As such, the higher age-standardized incidence rates of NETs in Singapore compared to Taiwan are possibly due to factors other than ethnicity alone, and additional genetic and sociogeographical factors including modifiable environmental factors ${ }^{14}$ and healthcare resource utilization ${ }^{2}$ should be evaluated in a further study.

Another secondary outcome studied was overall survival at 5 years and at 10 years. Our data have shown overall survival at 5 and 10 years to be 38.1 and $22.0 \%$, respectively. It is surprising that our overall survival results are lower than those reported by Taiwan (5-year survival at $50.4 \%$ for all NETs ${ }^{14}$ ), Norway (5-year survival at $50 \%$ for all $\mathrm{NETs}^{8}$ ), and North America (5-year survival for all NETs at $55 \%$ among whites and $59 \%$ among blacks $^{8}$ ), and this is likely to be driven by the primary tumor site, gender, advancing age in our population, and the stage at diagnosis.

This surfaces as a further area to be investigated in future studies, especially given that NETs are a heterogeneous group of tumors with several differing histological types, pathological grades, and a wide range of clinical behaviors. ${ }^{2}$ It would be beneficial to identify specific subgroups of NETs, which may potentially be more common in the local Singaporean population but which may simultaneously also suggest poorer prognosis and overall survival.

\section{Conclusion and Clinical Significance}

In conclusion, the age-standardized incidence of NETs in Singapore has demonstrated an almost fourfold increase over the last 22 years. This increase may be accounted for by the development of formal diagnostic classification systems for NETs and by advancements in the technology and availability of diagnostic tools. However, given the regional differences in the incidences as well as survival outcomes, further sociogeopolitical and genetic factors need to be investigated and further subgroup analyzes undertaken to identify prognosticating factors.

\section{References}

1. Dasari A, Shen C, Halperin D, et al. Trends in the incidence, prevalence, and survival outcomes in patients with neuroendocrine tumors in the United States. JAMA Oncol 2017;3(10):1335-1342. DOI: 10.1001/ jamaoncol.2017.0589.

2. Hallet J, Law $\mathrm{CH}$, Cukier $\mathrm{M}$, et al. Exploring the rising incidence of neuroendocrine tumors: a population-based analysis of epidemiology, metastatic presentation, and outcomes. Cancer 2015;121(4):589-597. DOI: 10.1002/cncr.29099.

3. Modlin IM, Lye KD, Kidd M. A 5-decade analysis of 13,715 carcinoid tumors. Cancer 2003;97(4):934-959. DOI: 10.1002/cncr.11105.

4. Fraenkel M, Kim M, Faggiano A, et al. Incidence of gastroenteropancreatic neuroendocrine tumours: a systematic review of the literature. Endocr Relat Cancer 2014;21(3):R153-R163. DOI: 10.1530/ERC-13-0125.

5. Ellis L, Shale MJ, Coleman MP. Carcinoid tumors of the gastrointestinal tract: trends in incidence in England since 1971. Am J Gastroenterol 2010;105(12):2563-2569. DOI: 10.1038/ajg.2010.341.

6. Yao JC, Hassan M, Phan A, et al. One hundred years after "carcinoid": epidemiology of and prognostic factors for Neuroendocrine tumors in 35,825 cases in the United states. J Clin Oncol 2008;26(18): 3063-3072. DOI: 10.1200/JCO.2007.15.4377.

7. Skuladottir $\mathrm{H}$, Hirsch FR, Hansen $\mathrm{HH}$, et al. Pulmonary neuroendocrine tumors: incidence and prognosis of histological subtypes. a population-based study in Denmark. Lung Cancer 2002;37(2):127-135. DOI: 10.1016/S0169-5002(02)00080-6.

8. Hauso O, Gustafsson BI, Kidd M, et al. Neuroendocrine tumor epidemiology: contrasting Norway and north America. Cancer 2008;113(10):2655-2664. DOI: 10.1002/cncr.23883.

9. Hemminki K, LiX. Incidence trends and risk factors of carcinoid tumors: a nationwide epidemiologic study from Sweden. Cancer 2001;92(8): 2204-2210. DOI: 10.1002/1097-0142(20011015)92:8<2204::AIDCNCR1564>3.0.CO;2-R.

10. Korse CM, Taal BG, van Velthuysen $M L$, et al. Incidence and survival of neuroendocrine tumours in the Netherlands according to histological grade: experience of two decades of cancer registry. Eur J Cancer 2013;49(8):1975-1983. DOI: 10.1016/j.ejca.2012.12.022.

11. Niederle MB, Hackl M, Kaserer K, et al. Gastroenteropancreatic neuroendocrine tumours: the current incidence and staging based on the WHO and European Neuroendocrine tumour society classification: an analysis based on prospectively collected parameters. Endocr Relat Cancer 2010;17(4):909-918. DOI: 10.1677/ ERC-10-0152.

12. Ito T, Tanaka M, Imamura M. Neuroendocrine tumor workshop J. [results of a nationwide survey of gastrointestinal tumors in Japan]. Nihon Geka Gakkai Zasshi 2008;109(3):128-132.

13. Ito $\mathrm{T}$, Tanaka $\mathrm{M}$, Sasano $\mathrm{H}$, et al. Preliminary results of a Japanese nationwide survey of neuroendocrine gastrointestinal tumors. J Gastroenterol 2007;42(6):497-500. DOI: 10.1007/s00535-007-2056-6.

14. Tsai HJ, Wu CC, Tsai CR, et al. The epidemiology of neuroendocrine tumors in Taiwan: a nation-wide cancer registry-based study. PLoS One 2013;8(4):e62487. DOI: 10.1371/journal.pone.0062487.

15. Cheema A, Weber J, Strosberg JR. Incidental detection of pancreatic neuroendocrine tumors: an analysis of incidence and outcomes. Ann Surg Oncol 2012;19(9):2932-2936. DOI: 10.1245/s10434-012-2285-7.

16. Modlin IM, Moss SF, Chung DC, et al. Priorities for improving the management of gastroenteropancreatic neuroendocrine tumors. J Natl Cancer Inst 2008;100(18):1282-1289. DOI: 10.1093/jnci/djn275.

17. Bosman FT, Carneiro F, Hruban RH, et al. WHO Classification of Tumours of the Digestive System. 4th edn, Lyon, France: International Agency for Research on Cancer (IARC); 2010. 